\title{
Lesión celular del miocardio y actividad de la ATPsintasa mitocondrial en ratas infectadas con una cepa colombiana de Trypanosoma cruzi
}

\author{
Dairo Alonso Rendón ${ }^{1}$, Carlos M. Genes ${ }^{2}$, Omar Triana ${ }^{2}$ \\ ${ }^{1}$ Facultad de Ciencias, Laboratorio de Biofísica, Universidad Nacional de Colombia sede Medellín, \\ Medellín, Colombia. \\ ${ }^{2}$ Grupo de Chagas, Instituto de Biología, Universidad de Antioquia, Medellín, Colombia.
}

Introducción. La enfermedad de Chagas es la principal causa de cardiomiopatía crónica en regiones endémicas de Latinoamérica. Alteraciones en el metabolismo energético mitocondrial cardiaco causadas por Trypanosoma cruzi pueden estar involucradas en el desarrollo de esta cardiomiopatía.

Objetivo. En este trabajo se investigó la lesión celular del miocardio de ratas durante una infección por la cepa colombiana Mg8 de Trypanosoma cruzi y la actividad de la ATPsintasa mitocondrial para correlacionar el daño al miocardio con el metabolismo energético mitocondrial.

Materiales y métodos. Se utilizaron grupos de cinco ratas, las cuales fueron infectadas con tripomastigotes. El curso de la infección se evaluó a nivel parasitológico, histopatológico y molecular. La actividad de la ATPsintasa mitocondrial del miocardio se evaluó tanto en las ratas infectadas con parásitos como en ratas control no infectadas.

Resultados. Se determinaron los días 26 y 60 post-infección como el punto de máxima parasitemia y como el punto de desaparición de los parásitos en sangre circulante, respectivamente. Los resultados histopatológicos y moleculares muestran que la cepa Mg8 tiene tropismo por el tejido cardiaco y causa una considerable lesión celular del miocardio de ratas tanto a 26 días (fase aguda) como a 60 días post-infección (fase crónica). A pesar de la lesión observada, no se encontró diferencia estadísticamente significativa en la actividad de la ATPsintasa de las ratas infectadas en estos días al comparar con sus respectivos controles. Conclusión. Estos resultados sugieren que el metabolismo energético mitocondrial no está alterado en la lesión celular del miocardio de las ratas, observada durante la infección con la cepa colombiana Mg8 de T. cruzi.

Palabras clave: Trypanosoma cruzi, enfermedad de Chagas, miocardiopatía chagásica, metabolismo energético, mitocondria.

Myocardial cellular damage and the activity of the mitochondrial ATP synthase in rats infected with a Colombian strain of Trypanosoma cruzi

Introduction. Chagas disease is the main cause of cardiomyopathy in endemic regions of Latin America. Alterations in the cardiac mitochondrial energy metabolism caused by Trypanosoma cruzi can be involved in the development of this cardiomyopathy during the course of Chagas disease.

Objective. The cellular injury of the rat myocardium was investigated in rats infected with the Colombian Mg8 strain of Trypanosoma cruzi. The activity of mitochondrial ATP synthase was measured to determine the relationship heart damage with the energy metabolism.

Materials and methods. Two groups of five rats each were infected with tripomastigotes, with 1 group of 6 rats serving as controls. The course of infection was characterized by parasitological, histopathological and molecular studies. The mitochondrial ATP synthase activity of the myocardium was evaluated in all rats.

Results. Peak parasitaemia (day 26 post infection) and the time of parasite clearance from circulating blood (day 60 post infection) were determined for acute and chronic phase models. 
The histopathological and molecular results showed that the Colombian Mg8 strain has tropism to the cardiac tissue and causes considerable cellular injury of the myocardium in rats during both phases. Despite the lesions observed in infected rats, no statistical difference in the activity of the mitochondrial ATPsynthase was observed between them and the non-infected rats.

Conclusion. Mitochondrial energy metabolism of the cardiomyocites does not appear to change during cellular injury of rat myocardium associated with infection by the Colombian Mg8 T. cruzi strain.

Key words: Trypanosoma cruzi, Chagas disease, Chagas cardiomyopathy, energy metabolism, mitochondria.

Trypanosoma cruzi, agente causal de la enfermedad de Chagas, afecta aproximadamente a 17 millones de personas de diferentes regiones de Centro y Suramérica, de las cuales probablemente entre el 20 y el 35\% desarrolla la enfermedad. En Colombia, la enfermedad de Chagas se encuentra ampliamente distribuída en las zonas rurales y se estima que el $5 \%$ de la población asentada en las zonas endémicas está infectada (1).

La cardiomiopatía es la afección más importante secundaria a esta enfermedad, ya que puede producir muerte súbita o discapacidad física, lo que representa grandes costos socioculturales y económicos. Para esclarecer la génesis de la cardiomiopatía chagásica se ha prestado gran atención a las alteraciones en el metabolismo energético mitocondrial del miocardio $(2,3)$. Las mitocondrias se consideran uno de los organelos más importantes de los cardiomiocitos, ya que en condiciones fisiológicas generan más del $90 \%$ de la energía necesaria para su funcionamiento (4). Además, pueden ser blanco de múltiples daños, producto de la respuesta inmunológica del hospedero a la infección (5). La importancia de las mitocondrias en el funcionamiento de las células cardiacas también se manifiesta en el hecho de que entre el 25 y $35 \%$ del volumen de los cardiomiocitos está ocupado por ellas. Es así como los eventos patofisiológicos que alteran la

\footnotetext{
Correspondencia:

Omar Triana Chávez, Calle 67 N. 53-108, Instituto de Biología, Universidad de Antioquia, Medellín, Colombia. Telefax: 2105622

otriana@matematicas.udea.edu.co

Recibido: 10/08/05; aceptado: 24/03/06
}

producción de energía por parte de las mitocondrias (síntesis del ATP), pueden desembocar en lesiones importantes del miocardio (6).

En la producción de energía en los cardiomiocitos juega un gran papel la enzima ATPsintasa mitocondrial, la cual cataliza la síntesis de ATP utilizando la energía almacenada en el potencial electroquímico de la membrana mitocondrial interna. Por su importancia, esta enzima mitocondrial se ha estudiado ampliamente en las últimas tres décadas, tanto en condiciones fisiológicas como patológicas (7-10). Uyemura et al. (2) y Vyatkina et al. (3) mostraron una disfunción en la síntesis de ATP por parte de la ATPsintasa mitocondrial del miocardio durante la infección con Trypanosoma cruzi utilizando mitocondrias aisladas.

Considerando que las cepas colombianas de $T$. cruzi tienen tropismo hacia el tejido cardiaco (11), es importante evaluar la posible lesión celular del miocardio durante una infección con estas cepas y la asociación de tal lesión con el metabolismo energético mitocondrial. En el presente trabajo se determinó la lesión celular del miocardio de ratas infectadas con una cepa colombiana de $T$. cruzi y se evaluó la actividad de la ATPsintasa mitocondrial en homogeneizados de este tejido.

\section{Materiales y métodos}

\section{Animales de laboratorio e infección con el parásito}

Se inocularon subcutáneamente ratas hembras de la cepa Wistar de aproximadamente 200 gramos con $2 \times 10^{5}$ tripomastigotes sanguíneos obtenidos de ratones Balb/c previamente irradiados con 450 rad de rayos gamma en un volumen total de sangre de entre 50 y $100 \mu$ l. 
Para la infección se usó la cepa colombiana Mg8, perteneciente al grupo I de Trypanosoma cruzi. Esta cepa se aisló en el año 2003 a partir del vector Triatoma dimidiata de origen silvestre procedente del departamento del Magdalena, y se mantuvo por repiques sucesivos cada siete días en medio de cultivo líquido LIT a $28^{\circ} \mathrm{C}(12)$ y por infección en ratones Balb/c. Los ensayos realizados en este estudio se iniciaron en el mismo año de aislamiento de la cepa, después de 18 pases por medio de cultivo y varios pases por ratón.

Todos los animales se mantuvieron de acuerdo a la reglamentación establecida por el Comité de Ética para la Experimentación con Animales de la Universidad de Antioquia.

\section{Caracterización de la infección}

Se utilizó un grupo de seis ratas para seguir el curso de la infección y determinar la fase aguda (máxima parasitemia en sangre circulante) y crónica de la enfermedad (desaparición de la parasitemia en sangre circulante). La evaluación de los animales infectados se realizó día por medio haciendo conteo de tripomastigotes sanguíneos en placa en un microscopio óptico con objetivo de 40X (13). Adicionalmente, se inocularon dos grupos de cinco ratas cada uno para determinar la actividad de la ATPsintasa mitocondrial y se hizo evaluación por reacción en cadena de la polimerasa (PCR) en la fase aguda y crónica de la enfermedad; así mismo, se inoculó un grupo de dos animales, para realizar los estudios histopatológicos tanto en la fase aguda como crónica de la enfermedad. Cada grupo de animales infectados tuvo un grupo control no infectado de igual número de animales mantenido en las mismas condiciones de laboratorio.

\section{Lesión celular del miocardio}

Para determinar la lesión celular del miocardio, a dos grupos de animales infectados y a sus respectivos controles se les extrajo el corazón a 26 y 60 dpi., e inmediatamente se fijó en formol al $10 \%$. Los corazones se seccionaron en su totalidad en cortes seriados transversales de 2 a $3 \mathrm{~mm}, y$ se deshidrataron empleando una serie de alcoholes de gradación ascendente $(50,70,80$ y
$100 \%$ ) y finalmente en xilol. Luego, los cortes se incluyeron en parafina y se cortaron con el micrótomo en secciones tisulares de tres a cinco micrómetros de espesor. Las muestras se tiñeron con hematoxilina-eosina para detectar la presencia del parásito y el nivel de inflamación del tejido, y con coloración de tricrómico para determinar el grado de fibrosis del tejido cardiaco.

\section{Extracción de ADN}

El ADN se extrajo a partir de una preparación de tejido cardiaco de animales infectados, utilizando el método de Salting Out (14), y en algunos casos el método de fenol-cloroformo (15). Las muestras de ADN se disolvieron en $50 \mathrm{ml}$ de agua destilada desionizada estéril a $37^{\circ} \mathrm{C}$ por cinco minutos (16) y se guardaron a $-20^{\circ} \mathrm{C}$.

\section{Detección del parásito por PCR}

Se utilizaron dos marcadores diferentes para detectar la presencia del parásito en los tejidos cardiacos a partir de una preparación de corazón empleada en la determinación de la actividad de la ATPsintasa mitocondrial: el ADN del cinetoplasto (kDNA) y el espaciador intergénico de los genes mini-exón. Para la amplificación de la región variable del kDNA se utilizaron los iniciadores $\$ 35$ y S36, que amplifican un fragmento de $330 \mathrm{pb}$ (17). La mezcla de reacción para PCR se llevó a cabo en un volumen final de $25 \mu \mathrm{l}$ que contenían 2,5 $\mu \mathrm{l}$ de ADN molde a una concentración de $50 \mathrm{ng} / \mu \mathrm{l}, 2 \mathrm{mM} \mathrm{MgCl}, 2 \mathrm{mM}$ dNTPs, $10 \mathrm{pmol} / \mu \mathrm{l}$ iniciadores, 0,05 unidades de Taq ADN polimerasa (Fermentas), buffer 10X (750mM Tris- $\mathrm{HCl} \mathrm{pH} 8,8 ; 200 \mathrm{mM}(\mathrm{NH} 4)_{2} \mathrm{SO}_{4}$ y $0,1 \%$ Tween 20$)$, y agua tridestilada estéril.

Los ciclos de la reacción se realizaron a una temperatura inicial de $94^{\circ} \mathrm{C}$ por tres minutos, seguida por 35 ciclos a $94^{\circ} \mathrm{C}$ por 45 segundos, a $63^{\circ} \mathrm{C}$ por 45 segundos y a $72^{\circ} \mathrm{C}$ por 45 segundos, y un último ciclo a $72^{\circ} \mathrm{C}$ por 10 minutos. El espaciador intergénico de los genes mini-exón se amplificó utilizando los iniciadores TC1, TC2 y TCC, los cuales amplifican un fragmento de 300 pb para el grupo T. cruzill y un fragmento de 350 pb para el grupo T. cruzil (18). La PCR se realizó en un volumen final de $25 \mu \mathrm{l}$ que contenían $2,5 \mu \mathrm{l}$ de ADN molde a una concentración de $50 \mathrm{ng} / \mu \mathrm{l}$, 
$1,5 \mathrm{mM} \mathrm{MgCl}, 10 \mathrm{pmol}$ de cada iniciador, $2 \mathrm{mM}$ dNTPs, 0,025 unidades de Taq DNA polimerasa, buffer 10X (750mM Tris- $\mathrm{HCl}(\mathrm{pH} 8,8) ; 200 \mathrm{mM}$ $(\mathrm{NH} 4)_{2} \mathrm{SO}_{4}$ y $0,1 \%$ Tween 20 ), y agua tridestilada estéril. Los ciclos de amplificación se realizaron a una temperatura inicial de $94^{\circ} \mathrm{C}$ durante un minuto, seguida de 27 ciclos a $94^{\circ} \mathrm{C}$ por 30 segundos, a $55^{\circ} \mathrm{C}$ por 30 segundos, a $72^{\circ} \mathrm{C}$ por 30 segundos y un ciclo final $a 72^{\circ} \mathrm{C}$ por 10 minutos. Los productos amplificados se detectaron en geles de agarosa al 1,5\% teñidos con bromuro de etidio a $0,5 \mathrm{mg} / \mu \mathrm{l}$ y se visualizaron con luz ultravioleta (15).

\section{Actividad de la ATPsintasa mitocondrial}

Los corazones de ratas sacrificadas por dislocación cervical se extrajeron rápidamente y se pusieron en solución de preparación del tejido a un $\mathrm{pH}$ de 7,5 entre 0 y $2^{\circ} \mathrm{C}(0,17 \mathrm{M} \mathrm{KCl}, 10 \mathrm{mM}$ EDTA, 0,1\% suero de albúmina bovina, $10 \mathrm{mM}$ Tris- $\mathrm{HCl}$ ) durante dos a tres minutos. Los corazones se pasaron por una prensa manual y con el tamizado se obtuvo un homogeneizado de tejido cardiaco ( $3,5 \mathrm{ml}$ solución $/ 1 \mathrm{~g}$ de tejido) utilizando un equipo Potter-Elvehjem a una velocidad angular de $700 \mathrm{rev} / \mathrm{min}$ durante dos a tres minutos y a una temperatura entre 0 y $2^{\circ} \mathrm{C}$. Finalmente, el homogeneizado obtenido se conservó entre 0 y $2^{\circ} \mathrm{C}$ hasta iniciar las mediciones. La actividad de la ATPsintasa mitocondrial se estimó por medio de un método potenciométrico (19), y se calculó según la siguiente fórmula: actividad de la ATPsintasa mitocondrial $=2 \mathrm{~m} \times 200 \mathrm{nmol} \mathrm{P}_{\mathrm{i}} / \mathrm{M} \times\left(\mathrm{h}_{1}+\mathrm{h}_{2}\right)$, donde $h_{1} y_{2}$ (en unidades de $m V$ ) son las alturas de los saltos en la señal de registro después de adicionar dos veces consecutivamente $200 \mathrm{nmol}$ de fosfato inorgánico, $\mathrm{m}$ (en unidades de $\mathrm{mV} / \mathrm{min}$ ) es la pendiente de la señal de registro después de adicionar ADP, $M$ (en unidades de $\mathrm{mg}$ ) es la cantidad de proteína total adicionada con la suspensión de tejido cardiaco; y $P_{i}$ es fosfato inorgánico (figura 1).

Brevemente, los homogeneizados de corazón de rata (1 mg de proteína total de homogeneizado de tejido cardiaco) fueron incubadas durante un minuto y 30 segundos con $5 \mathrm{mM}$ de glutamatomalato; después se realizaron las siguientes tres

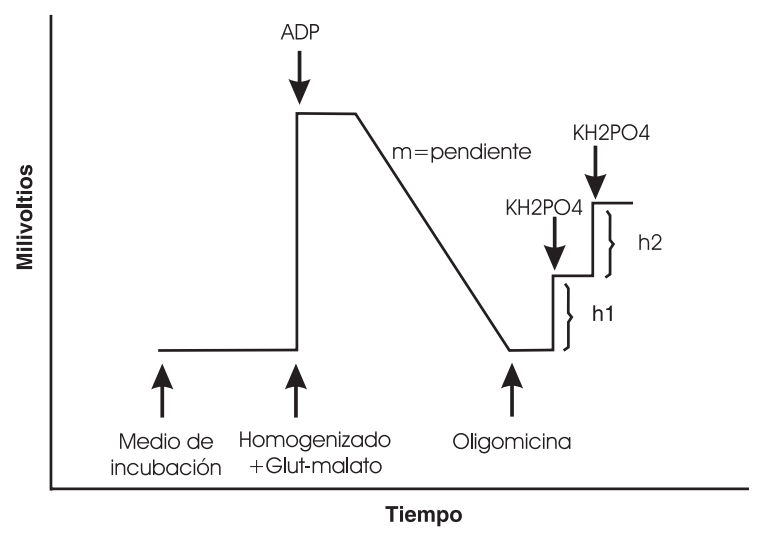

Figura 1. Curva típica en la determinación de la actividad de la ATPsintasa mitocondrial. En orden secuencial se adicionaron en los puntos indicados en las flechas $3,5 \mathrm{ml}$ medio de incubación, homogeneizado de corazón (1 mg de proteína total), $5 \mathrm{mM}$ glutamato-malato, $500 \mathrm{mM}$ de ADP, 0,8 $\mathrm{mg}$ oligomicina/mg de proteína total de homogeneizado, y por último, dos adiciones consecutivas de $200 \mathrm{nmol}$ de $\mathrm{KH}_{2} \mathrm{PO}_{4}$ cada una. Los homogeneizados de tejido cardiaco se incubaron durante $1 \mathrm{~min}$ y 30 segundos con $5 \mathrm{mM}$ de glutamato-malato antes de adicionar ADP. $\mathrm{El} \mathrm{KH}_{2} \mathrm{PO}_{4}$ se adiciona para calibrar la señal de registro.

adiciones: (i) 500 $\mu \mathrm{M}$ ADP; (ii) oligomicina, 0,8 $\mu \mathrm{g} / \mathrm{g}$ de proteína, y (iii) $200 \mathrm{nmol}$ de fosfato inorgánico $\left(\mathrm{KH}_{2} \mathrm{PO}_{4}\right)$ para calibrar la señal (figura 1). El alcohol que se adicionó con la oligomicina no superó el $0,1 \%$. La actividad de la ATPsintasa es equivalente a la velocidad de cambio de $\mathrm{pH}$ después de la adición de ADP calibrada con respecto al fosfato inorgánico y a la cantidad de proteína total adicionada con la suspensión de tejido cardiaco. El principio de este método potenciométrico radica en que la desaparición del fosfato inorgánico $\left(\mathrm{H}_{2} \mathrm{PO}_{4}{ }^{-}\right)$del medio debido a la síntesis de ATP por la ATPsintasa mitocondrial está asociada al cambio en el $\mathrm{pH}(\Delta \mathrm{pH})$ causado por la disociación del $\mathrm{H}_{2} \mathrm{PO}_{4}$ en $\mathrm{HPO}_{4}{ }^{2-}$ y H $\mathrm{H}^{+}\left(\mathrm{ATP}^{4-} \leftrightarrow \mathrm{ADP}^{3-+}\right.$ $\left.\mathrm{H}_{2} \mathrm{PO}_{4}-; \mathrm{H}_{2} \mathrm{PO}_{4}-\leftrightarrow \mathrm{HPO}_{4}{ }^{2-}+\mathrm{H}^{+}\right)$. La solución para la determinación de la actividad de la ATPsintasa mitocondrial fue de $250 \mathrm{mM}$ sacarosa, $5 \mathrm{mM}$ $\mathrm{KH}_{2} \mathrm{PO}_{4}, 2 \mathrm{mM}$ tris- $\mathrm{HCl}$, y 0,1 mM EDTA; $\mathrm{pH} 7,4$ $\left(\mathrm{HCl} / \mathrm{KOH}\right.$ a $\left.37^{\circ} \mathrm{C}\right)$. Las mediciones se realizaron en recipientes de vidrio bajo agitación magnética. El cambio en el pH se midió a $25^{\circ} \mathrm{C}$ utilizando un potenciómetro Corning modelo 12 de alta sensibilidad y rapidez conectado a un computador que visualiza la cinética del proceso. Las 
velocidades de síntesis del ATP se obtuvieron en nmol $P_{i} / \mathrm{mg}$ de proteína/min. La cuantificación de la proteína total en los homogeneizados de corazón se realizó por el método de Biuret (20), utilizando como patrón de calibración el suero de albúmina bovina.

\section{Análisis estadístico}

Para el análisis de los valores obtenidos en la actividad de la ATPsintasa mitocondrial se compararon cada uno de los grupos infectados con su respectivo control. Se utilizaron los programas Graph Pad Prism versión 2,0 y JMP versión 3.2.6. Se usó el $t$-test y se consideró $p<$ 0,05 como criterio de grupos significativamente diferentes. Los datos se presentaron como promedios \pm error estándar de la media (SEM). Las mediciones se realizaron por triplicado para cada animal sacrificado y luego se obtuvo el promedio de estos datos.

\section{Resultados}

\section{Caracterización de la infección}

El desarrollo de la enfermedad en las ratas infectadas con la cepa Mg8 estuvo marcado por la aparición de parásitos en sangre a los 11 días después de la infección (11 dpi). Se observó la presencia de dos picos de alta parasitemia: el primero en el día 26 (26 dpi), con aproximadamente $9,1 \times 10^{5}$ tripomastigotes por mililitro, y el segundo, en el día 40 pi, con aproximadamente $6,5 \times 10^{5}$ tripomastigotes por mililitro. La desaparición de la parasitemia fue estimada para el día 60 pi ( 60 dpi), que se consideró como el inicio de la fase crónica de la enfermedad (datos no mostrados).

\section{Lesión celular del miocardio}

El análisis histopatológico se realizó en tejido cardiaco de dos animales sacrificados a los 26 y 60 dpi y de sus respectivos controles. En los animales control se observaron fibras ordenadas, distribución normal del núcleo en el miocito y las células intersticiales se mostraban histológicamente sanas (figura 2A). En los animales sacrificados a los 26 dpi se observó miocarditis y no se encontró fibrosis (figura 2B). Se observó un importante infiltrado inflamatorio mononuclear, confluente con escasos seudoquistes (uno en un campo de alto poder) (figura 2C). En los animales sacrificados a los 60 dpi se observó infiltrado inflamatorio mononuclear a nivel intersticial alrededor de las fibras cardiacas (figura 3A), seudoquistes (tres en un campo de alto poder) (figura 3B), miocarditis, miocitolisis focal y áreas de fibrosis alrededor de algunas fibras cardiacas degeneradas (figuras $3 \mathrm{~A}$ y $3 \mathrm{C}$ ).

Dos de las seis ratas empleadas para la construcción de la curva de parasitemia murieron en los días 28 y 30 posterios a la infección. En estos animales se encontró un importante daño tisular en el tejido cardiaco. En el animal muerto a los 30 días pi se observaron abundantes fibras miocárdicas parasitadas con formación de
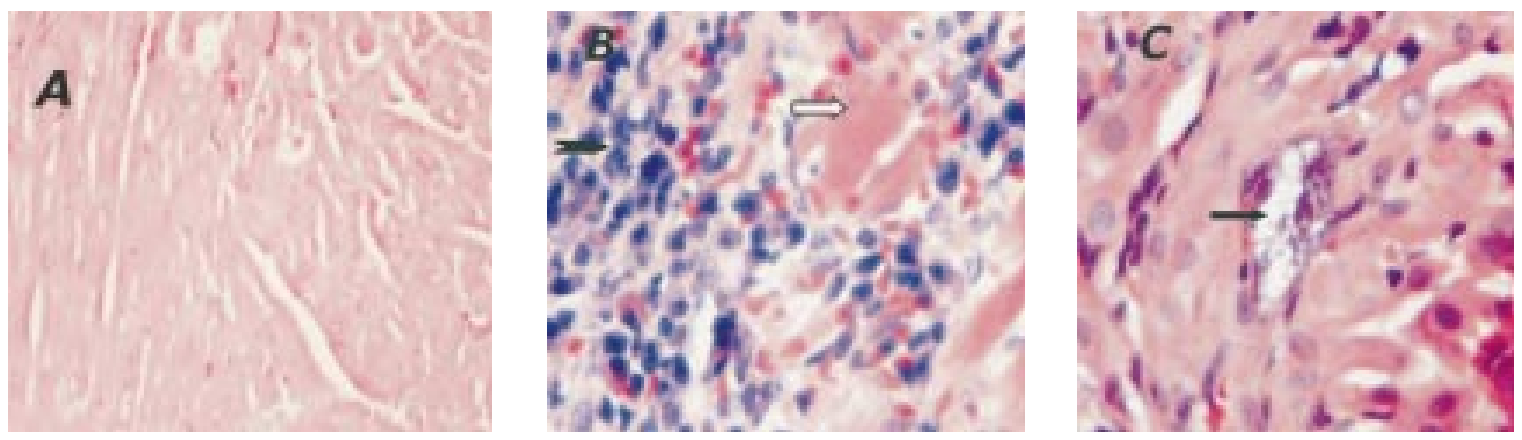

Figura 2. Corte histológico de tejido cardiaco de ratas infectadas con la cepa Mg8.

A. Rata control no infectada: no hay presencia de infiltrado inflamatorio, fibrosis o miocitolisis. B. Miocitólisis $(\Rightarrow)$ e infiltrado inflamatorio mononuclear observado en ratas a los 26 días pi (40X) (B). C. Seudoquiste de T. cruzi observado 26 días pi $(40 X)(\rightarrow)$. La tinción de los cortes fue realizada con hematoxilina-eosina. 

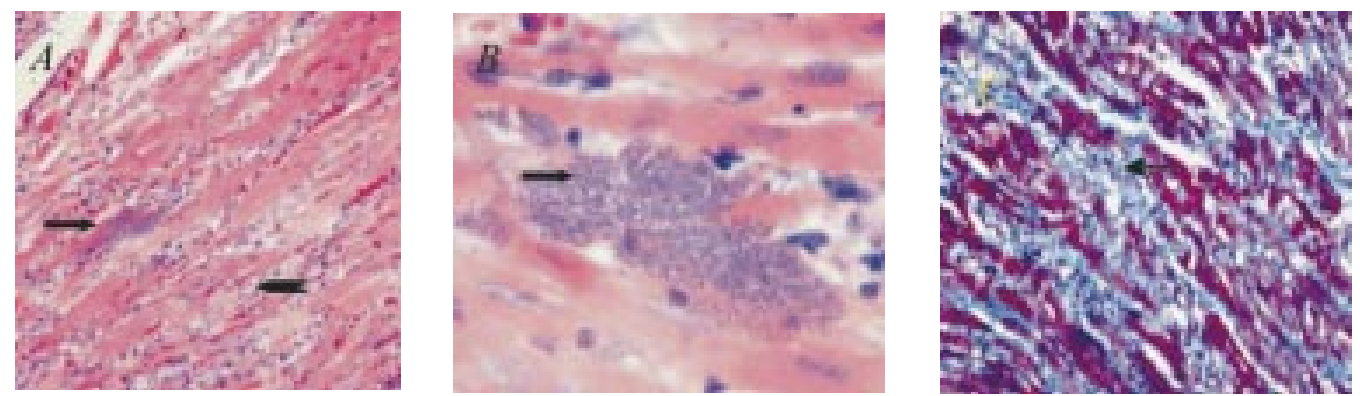

Figura 3. Corte de tejido cardiaco de rata infectada con la cepa Mg8 a los 60 días pi. A. Quiste de T. cruzi $(\rightarrow)$ e infiltrado inflamatorio mononuclear $(\square)(10 X)$. B. Nido de amastigotes $(\rightarrow)(40 X)$. C. Fibrosis $(\rightarrow)(40 X)$. La tinción de los cortes en las figuras $3 \mathrm{~A}$ y $3 \mathrm{~B}$ se realizó con hematoxilina-eosina. El corte de la figura $3 \mathrm{C}$ se tiñó con coloración de tricrómico.

seudoquistes intracelulares por la presencia de formas amastigotas de T. cruzi, reacción inflamatoria focal consistente en cúmulos de mononucleares, edema intersticial y fibrosis focal. En el animal muerto a los 28 días pi se encontró mayor reacción inflamatoria consistente en focos confluentes de células mononucleares con algunos polimorfonucleares neutrófilos alrededor de las fibras cardíacas, miocitolisis y edema. Se identificaron menos fibras cardiacas parasitadas, infiltrado mononuclear en el epicardio y no se observó fibrosis (datos no mostrados). En conclusión, los resultados muestran una grave lesión celular del miocardio en los corazones de estas ratas muertas durante el establecimiento de la infección.

\section{Análisis por PCR}

En el corazón de todos los animales infectados se observó la banda de 330 pb esperada para la región variable del marcador k-DNA de $T$. cruzi (figura 4A). De igual manera, se observó que todas las muestras amplifican la banda de $350 \mathrm{pb}$ para el gen mini-exón, característica del grupo I de $T$. cruzi (figura 4B).

\section{Actividad de la ATPsintasa mitocondrial}

La actividad de la ATPsintasa mitocondrial durante la infección con $T$. cruzise determinó en un grupo de cinco animales para el día 26 pi, que representa la fase aguda (figura $5 \mathrm{~A}$ ), y en otro grupo de cinco animales para el día 60 pi, que representa el inicio de la fase crónica (figura 5B). Cada grupo de animales infectados contaba con su respectivo grupo control $(n=5)$. La actividad de la ATPsintasa mitocondrial durante la infección al miocardio por T. cruzi en animales observada a los 26 pi no fue estadísticamente diferente a aquella obtenida para su respectivo control (figura $5 A)$. De igual forma, cuando se compararon las velocidades de síntesis de ATP para esta enzima en los animales y en sus respectivos controles a

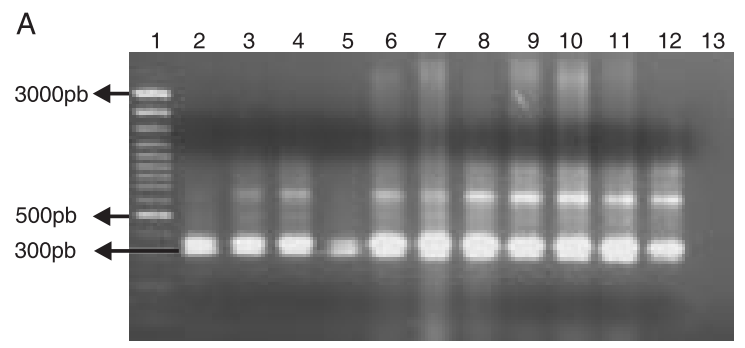

B

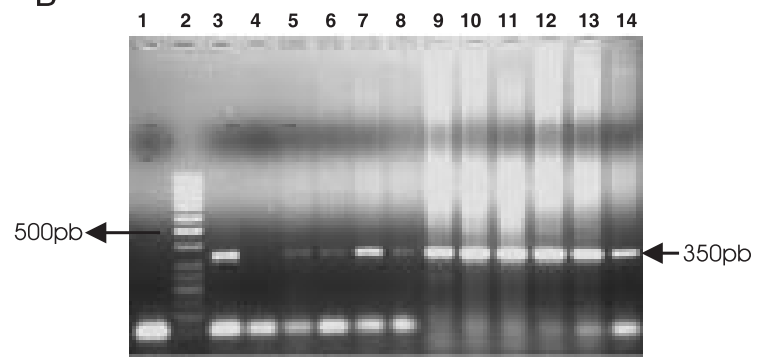

Figura 4. Amplificación por PCR de la región variable del kDNA (A) y la región intergénica del gen mini-exón (B). Se usó el ADN extraído de corazón de animales infectados a los días 26 y 60 después de la infección (pi). A. Carril 1: escalera de 100pb; carriles 2 a 6: muestras extraídas de tejido cardiaco de ratas a los 60 días pi; carriles 7 a 11: muestras extraídas de tejido cardiaco de ratas a los 26 días pi; carril 12: control positivo (ADN de cepa en cultivo); carril 13: control negativo. B. Carril 1: control negativo; carril 2: escalera de 100pb; carril 3: control positivo (ADN de cepa en cultivo); carril 4: control negativo (animal no infectado); carriles 5 a 9: muestras extraídas de tejido cardiaco de rata a los 60 días pi.; carriles 10 a 14: muestras extraídas de tejido cardiaco de rata a los 26 días pi. Gel de agarosa al $1,5 \%$ teñido con bromuro de etidio. 

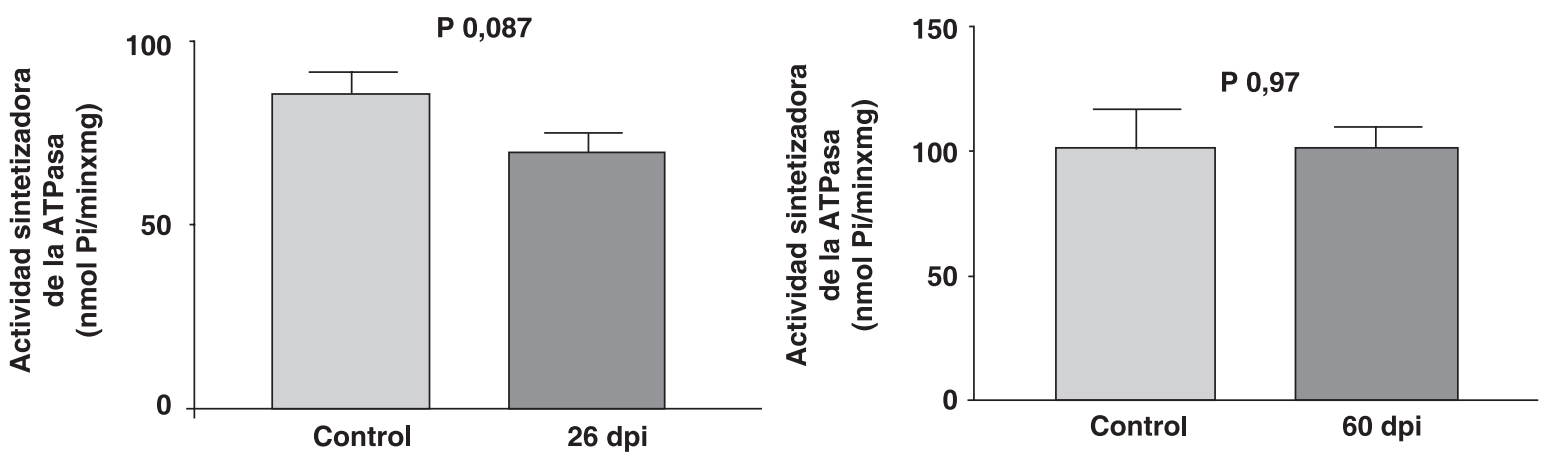

Figura 5. Actividad de la ATPsintasa mitocondrial en tejido cardiaco de rata a los 26 días pi (A) y 60 días pi (B) con T. cruzi. Los datos son los promedios de cinco mediciones independientes \pm SEM $(n=5) ; p<0,05$ fue el criterio de diferencias estadísticamente significativas. Cada medición independiente se realizó tres veces.

los $60 \mathrm{pi}$, no se evidenciaron diferencias estadísticamente significativas en la actividad de la ATPsintasa mitocondrial (figura 5B).

\section{Discusión}

La enfermedad de Chagas se presenta con una gran variedad de manifestaciones clínicas y es la mayor causa de miocarditis aguda y de cardiomiopatía crónica en las regiones endémicas de Latinoamérica $(21,22)$. Considerando que las cepas colombianas de $T$. cruzimuestran tropismo hacia el tejido cardiaco (11), es importante establecer si este tropismo está asociado con la lesión celular del miocardio en el hospedero. Para esto, la lesión celular del miocardio del hospedero se estudió en ratas infectadas con la cepa colombiana Mg8 de Trypanosoma cruzi aislada del vector Triatoma dimidiata silvestre en el mismo año de inicio de este estudio, lo que implica que conserva sus características naturales de virulencia y patogenicidad.

Los análisis histopatológicos (figuras 2 y 3 ) revelaron la capacidad de la cepa colombiana Mg8 para producir lesiones celulares en el miocardio del hospedero, características de la cardiomiopatía chagásica (22-25), lo que muestra que se está frente a una manifestación típica de la enfermedad de Chagas. Así, la presencia continua del parásito en las zonas de lesión del corazón puede ser importante en el origen de la cardiomiopatía chagásica (26).

Los resultados moleculares permiten asegurar que todos los organismos infectados tenían en su tejido cardiaco la presencia del parásito (ADN) o restos de éste, lo que garantiza que en todos los casos en que se evaluó la actividad de la ATPsintasa mitocondrial del miocardio, el parásito manifestó tropismo por el tejido cardiaco. Esto es importante ya que los tejidos de los animales infectados y usados para la determinación de la actividad de la ATPsintasa mitocondrial del miocardio no fueron sometidos a estudios histopatológicos debido a que todo el corazón se necesitó para preparar las suspensiones cardiacas.

Los resultados permiten concluir que la cepa Mg8 de Colombia produce importantes lesiones celulares en el tejido cardiaco, activación de la respuesta inmunológica a nivel de este órgano y presencia de las formas replicativas de $T$. cruzi, tanto a los 26 dpi como a los 60 dpi. Además, la cepa Mg8 es una cepa virulenta y altamente patogénica, hecho corroborado por la mortalidad antes de los 20 dpi que presentaron los ratones utilizados como fuente de tripomastigotes $(50 \%$ de mortalidad), y la presentada por las ratas utilizadas en la determinación de la curva de parasitemia ( $30 \%$ de mortalidad). Ello permite concluir que esta cepa generó en los individuos infectados un importante proceso degenerativo a nivel celular en el miocardio del hospedero, característico de la cardiopatía chagásica, lo que implica la presencia del parásito o de su ADN en el tejido cardiaco o en las células inflamatorias como un factor que probablemente contribuye en la producción de las lesiones y en la mortalidad observada en los hospederos estudiados (26). Es 
importante destacar que se observaron considerables lesiones celulares del miocardio del hospedero (figura 2) en un periodo relativamente inicial en la curva de parasitemia ( 26 dpi de la fase aguda), lo que permite concluir que en este inicio de la infección ya hay una manifestación clara de lesiones del miocardio.

Existen varias hipótesis sobre la patogénesis de la cardiomiopatía chagásica, como la neurogénica, la autoimmunidad (27-30), los problemas en la micro circulación del corazón $(31,32)$ y las alteraciones en el metabolismo energético mitocondrial del miocardio $(2,3)$. Esta última adquiere gran interés en el estudio de todas las patologías cardiovasculares, ya que más del $90 \%$ de la energía necesaria para el funcionamiento de los cardiomiocitos se produce en las mitocondrias. Por tal motivo era importante determinar si la lesión celular del miocardio observada a los 26 y 60 dpi con la cepa colombiana Mg8 de T. cruzi compromete al metabolismo energético mitocondrial del miocardio. En este proceso es crucial la actividad de la ATPsintasa mitocondrial, ya que esta enzima es en última instancia la encargada de sintetizar el ATP mitocondrial necesario para el buen funcionamiento de los cardiomiocitos. La actividad de la ATPsintasa mitocondrial representa en esencia la cantidad de ATP sintetizado por las mitocondrias contenidas en una unidad de tejido (en nuestro caso, $1 \mathrm{mg}$ de proteína total del homogeneizado de tejido cardiaco) por unidad de tiempo. Por ello, la actividad de la ATPsintasa mitocondrial es un criterio del funcionamiento del metabolismo energético mitocondrial del miocardio. Lo más usual es estudiar el metabolismo energético mitocondrial del miocardio en mitocondrias aisladas del tejido cardiaco o en partículas submitocondriales preparadas a partir de las mitocondrias aisladas. Sin embargo, se han reportado alteraciones estructurales y funcionales adicionales en las mitocondrias cuando éstas se aíslan de su ambiente natural (33). Dichas alteraciones adicionales pueden ser más marcadas cuando se usan tejidos isquémicos (34), como es el caso del tejido del corazón durante la infección con T. cruzi $(28,29)$. Por lo tanto, lo más conveniente para evitar estas posibles alteraciones adicionales de las mitocondrias en los estudios del metabolismo energético mitocondrial durante diferentes lesiones celulares del miocardio es el uso de homogeneizados rápidamente preparados a partir del tejido cardiaco $(35,36)$. En el presente trabajo fue crucial buscar las condiciones experimentales que permitieran diferenciar, usando homogeneizados de tejidos cardiacos infectados con parásitos, la actividad de la ATPsintasa mitocondrial tipo miocardio de la actividad de la ATPsintasa mitocondrial tipo parásito. La clave que permitió usar homogeneizados de tejido cardiaco en el presente trabajo radica que en $T$. cruzi el complejo I de la cadena transportadora de electrones no está funcionalmente presente (37). De esta forma, si usamos como substrato de respiración glutamato/malato, que pone a funcionar la cadena transportadora de electrones a partir del complejo I, en presencia de ADP sólo las mitocondrias que poseen este complejo (mitocondrias del corazón) podrán sintetizar ATP. Los resultados obtenidos (figura $5 \mathrm{~A}$ y $5 \mathrm{~B}$ ) indican que durante el proceso de inicio de la enfermedad de Chagas no hay alteración en la actividad enzimática de la ATPsintasa mitocondrial ni en el día 26 pi, fase aguda, ni en el día 60 pi, fase crónica en comparación con los respectivos controles; esto quiere decir que por cada unidad (en nuestro caso $1 \mathrm{mg}$ de proteína total de homogeneizado de tejido cardiaco) de tejido cardiaco infectado con la cepa colombiana Mg8 no hay déficit de cantidad de ATP mitocondrial (necesario para otras funciones celulares) por unidad de tiempo con respecto a los controles. Partiendo del hecho de que la actividad de la ATPsintasa mitocondrial es un criterio del metabolismo energético mitocondrial, estos resultados (figura $5 \mathrm{~A}$ y $5 \mathrm{~B}$ ) permiten afirmar que el metabolismo energético mitocondrial no está comprometido durante una infección con la cepa colombiana Mg8 a los 26 y 60 días pi, a pesar de las considerables lesiones celulares del miocardio observadas (figuras 2 y 3 ).

La no variación con respecto a cada control de la actividad de la ATPsintasa mitocondrial observada en homogeneizados de tejido cardiaco infectadas con la cepa colombiana Mg8 de T. cruzi no significa que uno de los complejos mitocondriales, 
o la fina sincronización entre estos, no se vea afectado negativamente durante la infección. Es posible que la cantidad de ATP mitocondrial producido por una sola mitocondria en la unidad de tiempo disminuya con respecto a las muestras no infectadas, pero que en un proceso de defensa el tejido cardiaco genere más mitocondrias por unidad de tejido infectado que su control para compensar la caída en la síntesis de ATP mitocondrial por una sola mitocondria. Este aspecto, por ahora simplemente especulativo, amerita futuras investigaciones.

Por último, es importante señalar que investigaciones previas reportan alteraciones en la actividad de la ATPsintasa mitocondrial durante una infección con $T$. cruzi, y con base en esto se concluye que durante la infección hay compromiso del metabolismo energético mitocondrial $(2,3)$. Es plausible que estas alteraciones negativas en la actividad de la ATPsintasa mitocondrial durante una infección del miocardio con $T$. cruzi sean producto de diferentes mecanismos de lesión celular del miocardio dependientes del tipo de cepa, o de alteraciones adicionales originadas durante la manipulación de las muestras biológicas $(33,34)$, dado que los investigadores utilizaron mitocondrias aisladas y partículas submitocondriales obtenidas a partir de tejidos isquémicos, como es el caso del tejido cardiaco durante una infección con $T$. cruzi $(28,29)$.

En conclusión, nuestros resultados sugieren que el metabolismo energético mitocondrial no está alterado en la lesión celular del miocardio de ratas infectadas con una cepa colombiana de $T$. cruzi perteneciente al grupo $T$. cruzi I. Es necesario realizar futuras investigaciones con cepas pertenecientes a los dos grupos de este parásito para entender las manifestaciones clínicas de la enfermedad de Chagas.

\section{Agradecimientos}

Al Laboratorio de Chagas de la Universidad de Antioquia y al Laboratorio de Biofísica de la Universidad Nacional de Colombia sede Medellín.

\section{Conflicto de intereses}

Los autores declaramos que no existe ningún conflicto de intereses.

\section{Financiación}

Esta investigación fue financiada por el CODI, proyecto CPT 0321 de la Universidad de Antioquia y por el DIME, proyecto 030802601 de la Universidad Nacional de Colombia, sede Medellín.

\section{Referencias}

1. Moncayo A. Chagas disease: current epidemiological trends after the interruption of vectorial and transfusional transmission in the Southern Cone countries. Mem Inst Oswaldo Cruz 2003;98:577-91.

2. Uyemura SA, Jordani MC, Polizello AC, Curti C. Heart $F_{0} F_{1}$-ATPase changes during the acute phase of Trypanosoma cruzi infection in rats. Mol Cell Biochem 1996;165:127-33.

3. Vyatkina G, Bhatia V, Gerstner A, Papaconstantinou J, Garg N. Impaired mitochondrial respiratory chain and bioenergetics during chagasic cardiomyopathy development. Biochim Biophys Acta 2004;1689:162-73.

4. Harris DA, Das AM. Control of mitochondrial ATP synthesis in the heart. Biochem J 1991;280:561-73.

5. Garg N, Popov VL, Papaconstantinou J. Profiling gene transcription reveals a deficiency of mitochondrial oxidative phosphorylation in Trypanosoma cruziinfected murine hearts: implications in chagasic myocarditis development. Biochim Biophys Acta 2003;1638:106-20.

6. Jennings RB, Hawkins HK, Lowe JE, Hill ML, Klotman S, Reimer KA. Relation between high energy phosphate and lethal injury in myocardial ischemia in the dog. Am J Pathol 1978; 92:187-214.

7. Boyer PD. The ATP synthase a splendid molecular machine. Annu Rev Biochem 1997;66:717-49.

8. Das Anibh M. Regulation of mitochondrial ATP synthase activity in human myocardium. Clin Sci 1998;94:499-504

9. Das Anibh M. Regulation of the mitochondrial ATPsynthase activity in health and disease. Mol Genet Metab 2003;79:71-82.

10. Senior AE, Nadanaciva S, Weber J. The molecular mechanism of ATP synthesis by Fo F1-ATP synthase. Biochim Biophys Acta 2002;1553:188-211.

11. Mejía AM, Triana O. Análisis por LSSP-PCR de la variabilidad genética de Trypanosoma cruzi en sangre y órganos de ratones. Biomédica 2005;25:76-86.

12. Camargo EP. Growth and differentiation in Trypanosoma cruzi. Origin of metacyclic trypanosomes in liquid media. Rev Inst Med Trop Sao Paulo 1964;12:93-100.

13. Brener $\mathbf{Z}$. Therapeutic activity and criterion of cure on mice experimentally infected with Trypanosoma cruzi. Rev Inst Med Trop Sao Paulo 1962;4:389-96. 
14. Miller SA, Dykes DD, Polesky HF. A simple salting out procedure for extracting DNA from human nucleated cells. Nucleic Acids Res 1988;16:1215.

15. Sambrook J, Fritsch EF, Maniatis T. Molecular cloning. A laboratory manual. 2 ed. Cold Spring Harbor, New York: Cold Harbor Laboratory Press;1989.

16. Maniatis T, Fritsch EF, Sambrook J. Molecular cloning. A laboratory manual. Cold Spring Harbor, New York: Cold Harbor Laboratory Press;1982.

17. Sturm N, Degrave W, Morel C, Simpson L. Sensitive detection and schizodeme classification of Trypanosoma cruzi cells by amplification of kinetoplast minicircle DNA sequences. Use in diagnosis of Chagas disease. Mol Biochem Parasitol 1989;33:205-14.

18. Fernández $\mathbf{O}$, Souto RP, Castro JA, Pereira JB, Fernández NC, Junqueira AC, et al. Brazilian isolates of Trypanosoma cruzi from humans and triatomines classified into two lineages using mini-exon and ribosomal RNA sequences. Am J Trop Med Hyg 1998;58:807-11.

19. Nishimura M, Ito T, Change B. Studies on bacterial photophosphorylation. III. A sensitive and rapid method of determination of photophosphorylation. Biochim Biophys Acta 1962;59:177-82.

20. Gornall AG, Bardawill CJ, David MM. Determination of serum proteins by means of the biuret reaction. $J$ Biol Chem 1949;177:751-66.

21. Chagas C. Estado sobre a morfologia e o ciclo evolutivo do Schizotrypanum cruzi, n. gen. sp. agente etiológico de nova entidade mórbida do homen. Mem Inst Oswaldo Cruz 1909;1:159-219.

22. Mukherjee S, Belbin TJ, Spray DC, lacobas Da, Weiss LM, Kitsis RN, et al. Microarray analysis of changes in gene expression in a murine model of chronic chagasic cardiomyopathy. Parasitol Res 2003;91:187-96.

23. Moreira da Silva A, Ramirez Le, Vargas $\mathbf{M}$, Chapadeiro E, Brener Z. Evaluation of the rabbit as a model for Chagas disease - II. Histopatologic studies of the Herat, digestive tract and skeletal muscle. Mem Inst Oswaldo Cruz 1996;91:199-206.

24. de Oliveira JS, Bestetti RB, Soares EG, Marin Nieto JA. Ajmaline-induced electrocardiographic changes in chronic Trypanosoma cruzi-infected rats. Trans R Soc Trop Med Hyg 1986;80:415-9.

25. Acosta AM, Santos-Buch CA. Autoimmune myocarditis induced by Trypanosoma cruzi. Circulation 1985;71:1255-61.
26. Jones EM, Colley DG, Tostes S, Lopes ER, VnencakJones CL, McCurley TL. Amplification of a Trypanosoma cruzi DNA sequence from inflammatory lesions in human chagasic cardiomyopathy. Am J Trop Med Hyg 1993;48:348-57.

27. Köberle F. Chagas' disease and Chagas' syndroms: the pathology of American trypanosomiasis. Adv Parasitol 1968;6:63-116.

28. Teixeira AR. Patogenia da doença de Chagas. J Bras Med 1980;38:23-33

29. Oliveira JS. A natural human model for intrinsic heart nervous system denervation: Chagas' cardiopathy. Am Heart J 1985;110:1092-8.

30. Junqueira Junior LF, Beraldo PS, Chapadeiro E, Jesus PC. Cardiac autonomic dysfunction and neuroganglionitis in a rat model of chronic Chagas' disease. Cardiovasc Res 1992;26:324-9.

31. Rossi MA, Carobrez SG. Experimental Trypanosoma cruzi cardiomyopathy in BALB/c mice histochemical evidence of hypoxic changes in the myocardium. $\mathrm{Br} \mathrm{J}$ Exp Pathol 1985;66:155-60.

32. Rossi MA. Microvascular changes as a cause of chronic cardiomyopathy in Chagas's disease. Am Heart J 1990;20:233-6.

33. Nohl H, Gille L, Stanick K. Intracellular generation of reactive oxygen species by mitochondria. Biochem Pharmacol 2005;69:719-23.

34. Veksler VI, Kuznetsov AV, Sharov VG, Kapelko VI, Saks VA. Mitochondrial respiratory parameters in cardiac tissue: a novel method of assessment by using saponin-skinned fibers. Biochim Biophys Acta 1987;892:191-6.

35. Rendon DA, Lopez LF. Mitochondrial oligomycinsensitive ATPase during isoproterenol-induced cell injury of myocardium. Arch Inst Cardiol Mex $2000 ; 70: 130-5$

36. Rendon DA, Lopez LF. Activation of mitochondria oxidative phosphorylation during (+/-)-isoproterenolinduced cell injury of myocardium. Arch Cardiol Mex 2001;71:13-9.

37. Denicola Seoane A, Rubbo H, Prodanov E, Turrens J. Succinate-dependent metabolism in Trypanosoma cruzi epimastigotes. Mol Biochem Parasitol 1992;54: 43-50. 